\title{
Change impact analysis of complex product using an improved three-parameter interval grey relation model
}

\author{
Yang, W.M. ${ }^{a}$, Li, C.D. ${ }^{a,}{ }^{,}$, Chen, Y.H. ${ }^{b}$, Yu, Y.Y. ${ }^{a}{ }^{, *}$ \\ ${ }^{a}$ School of Management Jinan University, Guangzhou, P.R. China \\ ${ }^{b}$ International Business School, Jinan University, Zhuhai, P.R. China
}

\begin{abstract}
A B S T R A C T
Change impact evaluation of complex product plays an important role in controlling change cost and improving change efficiency of engineering change enterprises. In order to improve the accuracy of engineering change impact evaluation, this paper introduces three-parameter interval grey number to evaluate complex products according to the data characteristics. The linear combination of BWM and Gini coefficient method is used to improve the three-parameter interval grey number correlation model. It is applied to the impact evaluation of complex product engineering change. This paper firstly constructs a multi-stage complex network for complex product engineering change. Then the engineering change impact evaluation index system is determined. Finally, a case analysis was carried out with the permanent magnet synchronous centrifugal compressor in a large permanent magnet synchronous centrifugal unit to verify the effectiveness of the proposed method.
\end{abstract}

\author{
ARTICLE INFO \\ Keywords: \\ Manufacturing; \\ Engineering; \\ Complex product; \\ Change impact analysis; \\ Three-parameter interval grey \\ number; \\ Grey relational model; \\ BWM method (best-worst model); \\ Gini weighting method \\ *Corresponding author: \\ licd@jnu.edu.cn \\ (Li, C.D.) \\ 3035905183@qq.com \\ (Yu, Y.Y.) \\ Article history: \\ Received 10 April 2021 \\ Revised 23 May 2021 \\ Accepted 5 June 2021

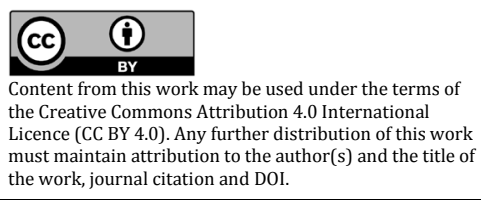

\section{References}

[1] Yang, D., Sun, Y., Wu, K. (2020). Assembly reliability modelling technology using function decomposing and LSSVM, International Journal of Simulation Modelling, Vol. 19, No. 2, 334-345, doi: 10.2507/IJSIMM19-2-C09.

[2] Awaga, A.L., Xu, W., Liu, L., Zhang, Y. (2020). Evolutionary game of green manufacturing mode of enterprises under the influence of government reward and punishment, Advances in Production Engineering \& Management, Vol. 15, No. 4, 416-430, doi: 10.14743/apem2020.4.375.

[3] Li, H.-Y., Xu, W., Cui, Y., Wang, Z., Xiao, M., Sun, Z.-X. (2020). Preventive maintenance decision model of urban transportation system equipment based on multi-control units, IEEE Access, Vol. 8, 15851-15869, doi: 10.1109/ACCESS.2019.2961433.

[4] Baynal, K., Sari, T., Akpinar, B. (2018). Risk management in automotive manufacturing process based on FMEA and grey relational analysis: A case study, Advances in Production Engineering \& Management, Vol. 13, No. 1, 6980, doi: 10.14743/apem2018.1.274.

[5] Sterpin Valic, G., Cukor, G., Jurkovic, Z., Brezocnik, M. (2019). Multi-criteria optimization of turning of martensitic stainless steel for sustainability, International Journal of Simulation Modelling, Vol. 18, No. 4, 632-642, doi: 10.2507/IJSIMM18(4)495. 
[6] Ocampo, L.A., Himang, C.M., Kumar, A., Brezocnik, M. (2019). A novel multiple criteria decision-making approach based on fuzzy DEMATEL, fuzzy ANP and fuzzy AHP for mapping collection and distribution centers in reverse logistics, Advances in Production Engineering \& Management, Vol. 14, No. 3, 297-322, doi: 10.14743/apem2019. $\underline{3.329}$.

[7] Cica, D., Caliskan, H., Panjan, P., Kramar, D. (2020). Multi-objective optimization of hard milling using taguchi based grey relational analysis, Tehnički Vjesnik - Technical Gazette, Vol. 27, No. 2, 513-519, doi: 10.17559/TV20181013122208.

[8] Liu, D., Hu, B., Ding, Z., Kaisarb, E.I. (2020). Method of group decision making with interval grey numbers based on grey correlation and relative close degree, Tehnički Vjesnik - Technical Gazette, Vol. 27, No. 5, 1579-1584, doi: $10.17559 / \mathrm{TV}-20200601165833$.

[9] Xiao, Y., Li, C., Song, L., Yang, J., Su, J. (2021). A multidimensional information fusion-based matching decision method for manufacturing service resource, Vol. 9, 39839-39851, doi: 10.1109/ACCESS.2021.3063277.

[10] Cheng, H., Chu, X. (2012). A network-based assessment approach for change impacts on complex product, Journal of Intelligent Manufacturing, Vol. 23 No. 4, 1419-1431, doi: 10.1007/s10845-010-0454-8.

[10] Ahmad, N., Wynn, D.C., Clarkson, P.J. (2013). Change impact on a product and its redesign process: A tool for knowledge capture and reuse, Research in Engineering Design, Vol. 24, No. 3, 219-244, doi: 10.1007/s00163012-0139-8.

[11] Chen, C.-Y., Liao, G.-Y., Lin, K.-S. (2015). An attribute-based and object-oriented approach with system implementation for change impact analysis in variant product design, Computer-Aided Design, Vol. 62, 203-217, doi: 10.1016/i.cad.2014.11.006.

[12] Maâzoun, J., Bouassida, N., Ben-Abdallah, H. (2016). Change impact analysis for software product lines, Journal of King Saud University-Computer and Information Sciences, Vol. 28, No. 4, 364-380, doi: 10.1016/j.jksuci. 2016.01. $\underline{005}$.

[13] Gong, X.Q., Wu, B., Wu, F. (2021). Research on the impact of green product packaging design on ecological environment, Fresenius Environmental Bulletin, Vol. 30, No. 4, 3228-3232.

[14] Zheng, Y.-J., Yang, Y., Zhang, N. (2020). A model for assessment of the impact of configuration changes in complex products, Journal of Intelligent Manufacturing, Vol. 31, No. 2, 501-527, doi: 10.1007/s10845-018-01461-w.

[15] Li, Y., Zhao, W. (2014). An integrated change propagation scheduling approach for product design, Concurrent Engineering, Vol. 22, No. 4, 347-360, doi: 10.1177/1063293X14553809.

[16] Ma, S., Jiang, Z., Liu, W. (2016). Evaluation of a design property network-based change propagation routing approach for mechanical product development, Advanced Engineering Informatics, Vol. 30, No. 4, 633-642, doi: 10.1016/i.aei.2016.08.002.

[17] Maldini, I., Stappers, P.J., Gimeno-Martinez, J.C., Daanen, H.A.M. (2019). Assessing the impact of design strategies on clothing lifetimes, usage and volumes: The case of product personalisation, Journal of Cleaner Production, Vol. 210, 1414-1424, doi: 10.1016/i.jclepro.2018.11.056.

[18] Zhang, N., Yang, Y. (2019). Change impact analysis of complex mechanical product based on complex network theory, Journal of Physics: Conference Series, Vol. 1187, No. 3, Article No. 032099, doi: 10.1088/1742-6596/ $1187 / 3 / 032099$.

[19] Maurya, S., Mougenot, C., Takeda, Y. (2021). Impact of mixed reality implementation on early-stage interactive product design process, Journal of Engineering Design, Vol. 32, No. 1, 1-27, doi: 10.1080/09544828.2020. 1851662.

[20] Palumbo, E., Soust-Verdaguer, B., Llatas, C., Traverso, M. (2020). How to obtain accurate environmental impacts at early design stages in BIM when using environmental product declaration. A method to support decisionmaking, Sustainability, Vol. 12, No. 17, Article No. 6927, doi: 10.3390/su12176927.

[21] Li, R., Yang, N., Zhang, Y., Liu, H. (2020). Risk propagation and mitigation of design change for complex product development (CPD) projects based on multilayer network theory, Computers \& Industrial Engineering, Vol. 142, Article No. 106370, doi: 10.1016/i.cie.2020.106370.

[22] Gao, Y., Li, D. (2018). UAV swarm cooperative situation perception consensus evaluation method based on threeparameter interval number and heronian mean operator, IEEE Access, Vol. 6, 73328-73340, doi: 10.1109/ ACCESS.2018.2882409.

[23] He, X., Li, Y., Qin, K. (2021). On a new distance measure of three-parameter interval numbers and its application to pattern recognition, Soft Computing, Vol. 25, 8595-8607, doi: 10.1007/s00500-021-05741-1.

[24] Li, Y., Zhu, S., Guo, S.-D. (2016). Multi-attribute grey target decision method with three-parameter interval grey number, Grey Systems: Theory and Application, Vol. 6, No. 2, 270-280, doi: 10.1108/GS-05-2016-0010.

[25] Nozari, H., Jafari-Eskandari, M., Kamfirozi, M.H., Mozafari, A. (2014). Using numerical taxonomy and combined bulls-eye-shapley weighting method in order to ranking websites of Iranian universities by three-parameter interval gray numbers, Arabian Journal for Science and Engineering, Vol. 39, 3299-3305, doi: 10.1007/s13369-0130881-X.

[26] Xu, L.-B., Li, X.-S., Shao, J.-K. Wang, K.-J. (2018). Extension dependent degree method with mapping transformation for three-parameter interval number decision making, Mathematical Problems in Engineering, Vol. 2018, Article ID 1831086, doi: 10.1155/2018/1831086.

[27] Li, Y., Zhang, D.X., Liu, B. (2019). Multi-attribute decision-making method based on cosine similarity with threeparameter interval grey number, Journal of Grey System, Vol. 31, No. 3, 45-58.

[28] Yin, Y., Ren, Q. (2018). Studying the representative volume of concrete using the entropy weight-grey correlation model, Magazine of Concrete Research, Vol. 70, No. 15, 757-769, doi: 10.1680/jmacr.17.00263.

[29] Liu, H., Dong, Y., Wang, F. (2020). Gas outburst prediction model using improved entropy weight grey correlation analysis and IPSO-LSSVM, Mathematical Problems in Engineering, Vol. 2020, Article ID 8863425, doi: 10.1155/ $\underline{2020 / 8863425}$. 
[30] Gu, Q., Wang, R., Ju, C. (2020). Evaluation path selection of opening-up level of chinese coastal cities based on entropy weight-topsis-grey correlation: From researches on ten coastal cities, Journal of Coastal Research, Vol. 115, No. 1, 636-640, doi: 10.2112/JCR-SI115-168.1.

[31] Li, B., Zhu, X. (2019). Grey relational decision making model of three-parameter interval grey number based on AHP and DEA, Grey Systems: Theory and Application, Vol. 10, No. 1, 25-37, doi: 10.1108/GS-10-2018-0049.

[32] Li, Y., Zhang, D. (2020). Multi-attribute group grey target decision-making method based on three-parameter interval grey number, Journal of Grey System, Vol. 32, No. 3, 96-109.

[33] Behzad, M., Zolfani, S.H., Pamucar, D., Behzad, M. (2020). A comparative assessment of solid waste management performance in the Nordic countries based on BWM-EDAS, Journal of Cleaner Production, Vol. 266, Article No. 122008, doi: $10.1016 /$ j.jclepro.2020.122008.

[34] Zhang, D., Shen, J., Liu, P., Zhang, Q., Sun, F. (2020). Use of fuzzy analytic hierarchy process and environmental gini coefficient for allocation of regional flood drainage rights, International Journal of Environmental Research and Public Health, Vol. 17, No. 6, Article ID 2063, doi: 10.3390/ijerph17062063. 


\section{APEM}

\title{
Analiza vpliva sprememb kompleksnega izdelka $z$ uporabo izboljšanega sivega relacijskega modela $\mathrm{z}$ intervalom treh parametrov
}

\author{
Yang, W.M. ${ }^{a}$, Li, C.D. ${ }^{\mathrm{a},{ }^{*},}$, Chen, Y.H. ${ }^{\mathrm{b}}$, Yu, Y.Y. ${ }^{\mathrm{a},{ }^{*}}$ \\ aSchool of Management Jinan University,Guangzhou, P.R. China \\ bInternational Business School, Jinan University, Zhuhai, P.R. China
}

\section{POVZETEK}

Za podjetja, ki vpeljujejo inženirske spremembe ima vrednotenje vpliva sprememb pri kompleksnih izdelkih pomembno vlogo pri nadzoru stroškov sprememb in izboljšanju učinkovitosti sprememb. Da bi izboljšali natančnost ocene vpliva inženirskih sprememb, je uveden sivi relacijski model z intervalom treh parametrov za oceno kompleksnih izdelkov glede na značilnosti podatkov. Linearna kombinacija metod BWM in Ginijevega koeficienta se uporablja za izboljšanje sivega relacijskega modela $\mathrm{z}$ intervalom treh parametrov. Uporablja se za vrednotenje vpliva kompleksnih inženirskih sprememb izdelka. Ta članek najprej vzpostavi večstopenjsko kompleksno omrežje za kompleksno inženirsko spremembo izdelka. Nato se ovrednoti sistem kazalnikov s katerimi se oceni vpliv inženirskih sprememb. Da bi preverili učinkovitost predlagane metode je bila na koncu izvedena študija primera s sinhronim centrifugalnim kompresorjem s trajnimi magneti v veliki sinhroni centrifugalni enoti s trajnimi magneti.

\section{PODATKI O ČLANKU}

Ključne besede:

Izdelava;

Inženiring;

Kompleksni izdelek;

Analiza vpliva sprememb;

Sivo število z intervalom treh pa-

rametrov;

Sivi relacijski model;

Metoda BWM (najboljši-najslabši

model);

Ginijeva metoda uteževanja

*Kontaktna oseba:

licd@jnu.edu.cn

(Li, C.D.)

3035905183@qq.com

(Yu, Y.Y.)

Zgodovina članka:

Prejet 10. aprila 2021

Popravljen 23. maja 2021

Sprejet 5. junija 2021

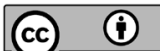

Content from this work may be used under the terms of the Creative Commons Attribution 4.0 International Licence (CC BY 4.0). Any further distribution of this work must maintain attribution to the author(s) and the title of the work, journal citation and DOI. 